\title{
Automatic Iris Segmentation Using Active Near Infra Red Lighting
}

\author{
Carlos H. Morimoto Thiago T. Santos Adriano S. Muniz \\ Departamento de Ciência da Computação - IME/USP \\ Rua do Matão, 1010, São Paulo, SP, Brazil \\ \{hitoshi, thsant, saturno\}@ime.usp.br
}

\begin{abstract}
This paper introduces a fast, robust and accurate iris segmentation technique based on active lighting. The geometry of the light sources, a single camera and the eye facilitates the detection of the pupil and the automatic selection of the most appropriate image for biometric identification from the video stream, minimizing the effects of noise, distortion and occlusion during the image acquisition process. Two near infrared (NIR) light sources (that are invisible to the human eye) are synchronized with the video signal. One of the light sources is placed near the optical axis of the camera, and generates a bright pupil image. The second light is placed off-axis, generating dark pupil images. These two images can be easily combined to segment the pupil region, and the corneal reflection of the light sources can be used to select best quality images. The pupil position is then used to segment the iris and eyelids using a coarse-to-fine strategy. Experimental results with a real-time prototype show the quality of the iris segmentation.
\end{abstract}

\section{Introduction}

The iris, the color part of the eye, is one of the best biometric feature for identification and authentication due to several properties, such as, it presents patterns that are very stable throughout a person's lifetime, the patterns are quite unique (even identical twins do not share the same iris pattern because it is a phenotypic feature) and reliable (it is present in practically every one, and is not susceptible to loss, theft or compromise). Besides, the iris pattern acquision process is very flexible and non-invasive (only a simple picture of the eye is required), allowing this technology to be easily combined with other biometric features, such as voice and fingerprints.

Flom and Safir were the first to suggest the use of iris for recognition in 1987 [6], but it seems that they never presented a working system. Figure 1 shows the block diagram of a general iris recognition system. The iris is first seg- mented and features are extracted from the iris region. The input image can be identified or authenticated by matching the extracted features with patterns stored in an iris database.

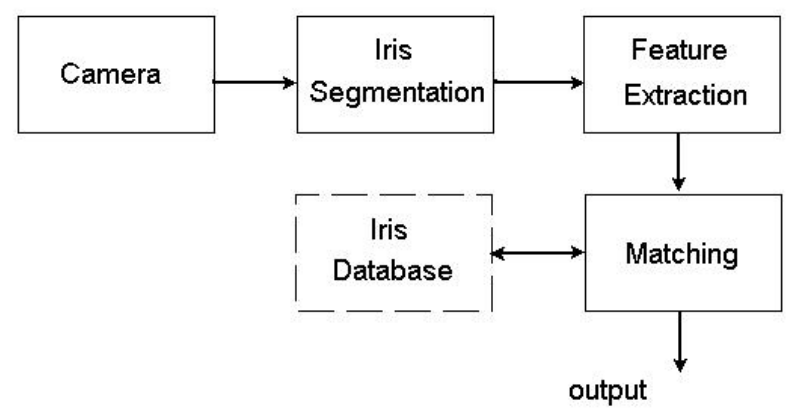
Figure 1. Block diagram of a general iris
recognition system.

Probably the two most influential and tested methods for automatic iris recognition are those of Daugman [3] and Wildes et al. [9]. Most of the commercial systems available today are based on Daugman's work. The iris segmentation module of Daugman's system uses integro-differential operators that behave like circle edge detectors, to estimate the inner an outer boundaries of the iris. A similar procedure is used to detect the upper and lower eyelid boundaries. Using quadrature 2D Gabor filters, Daugman extracts the phase information of the iris pattern. Patches of the phase information are encoded to represent the quadrant of the complex plane each phasor lies, setting two bits of information. This process is repeated all across the iris with many wavelet sizes, frequencies, and orientations, to extract 1024 phasors, or 2048 bits ( 256 bytes). A test of statistical independence is implemented by a simple XOR operator, to detect disagreements between bits of the iris patterns being compared. In a perfect match, no disagreements are detected.

Wildes et al. [9] use Hough transform to segment the iris 
boundaries and eyelids. The iris pattern is represented by a Laplacian pyramid. Normalized correlation is used to determine whether the input image matches the models stored in the iris database.

Following these two systems, several others have been suggested. For example, Boles and Boashash [2] first find the pupil and iris using edge detection techniques. Then they compute zero-crossings of the wavelet transform at various resolution levels over concentric circles on the iris, and the resulting one-dimensional signals are compared with model features using different dissimilarity functions. Zhu et al. [12] also use integral-differential operators to segment the iris. The iris pattern is decomposed into multiple bands using a two-dimensional Gabor transform, and the means and variances of all bands are used for shift invariant feature representation. The iris recognition home page (http://www.iris-recognition.org) presents several other links to patents, vendors and academic works.

Though it is one of the latest emerging technologies in biometrics, proofs of the maturity of iris recognition technology (IRT) are the pilot programs at ATMs in England and the USA; and employee and passenger authentication at airports in several countries in Europe. But despite the several advantages of IRT, according to the International Biometric Group, they are still somewhat offset by the level of training required to use the system effectively. To illustrate this point, the iris recognition system described by Wildes et al. [9] provides a reticle to help the operator to place his/her eye in the correct position. Two square contours centered around the camera lens are placed in different planes. At arbitrary positions, the user sees the two squares, but when the eye is in the appropriate position, the squares overlap and appear as one. Holding the eye at this position, the user activates the image capture by pressing a button.

The positioning of the user is particularly difficult using low cost off-the-shelf components such as fixed focus micro cameras or webcams. Because the identification system needs a high resolution close up image of the eye, the eye must be located within a small region, typically about $10 \mathrm{~cm}^{3}$, in order to obtain a good quality image. Besides positioning, other problems may arise during image acquisition such as occlusion of the iris by the eyelids, strong specular reflections on the iris region, bad imaging or illumination conditions such as out of focus or low contrast, etc.

This paper presents a robust, efficient, easy to use and low cost alternative to iris segmentation. The technique is based on a robust dual NIR (near infrared) differential lighting scheme for pupil detection. The NIR lighting guarantees good illumination conditions during image acquisition and the means to compute a particular eye-camera configuration that can be used to select good images of the iris. Once a good image of the pupil is obtained, the segmentation of the outer circle of the iris and the eyelids is greatly facilitated, as shown by our experimental results.

The rest of this paper is organized as follows. The next section introduces the basic operation of our vision based automatic iris segmentation system. Section 3 discusses some implementation issues and experimental results, and Section 4 concludes the paper.

\section{Pupil, Iris, and Eyelid Segmentation}

Figure 2 shows the major components of the human eye. The eye has an approximately spherical shape with a radius of about $12 \mathrm{~mm}$ [10]. The external parts of the eye that are visible in the eye socket are the sclera (the white part of the eye), the iris (the color part of the eye), and the pupil located in the center of the iris. The cornea is a protective transparent membrane, void of blood vessels, that protrudes toward the front of the eye, and covers the iris. The cornea is constantly lubricated by blinking the eyelids which, in their normal position, partially covers the iris.

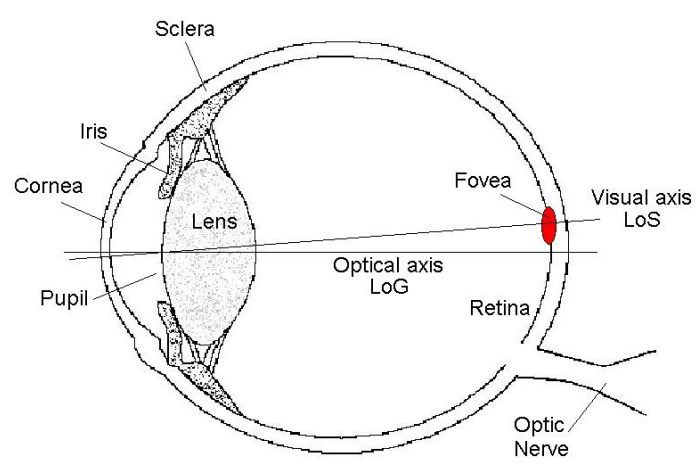

Figure 2. Structure of the human eye

The iris controls the pupil size by the action of its dilator ad sphincter muscles. Its complex fibrillous pattern is due to the elastic connective tissue that constitute these muscles. The eye structure is built from the 3rd to about the 8th month of gestation, although changes in pigmentation can occur during the first year of life. The texture pattern of a human iris is not a genetic feature, as the reader might confirm by carefully examining the textures of their left and right eyes (which are, off course, genetically identical). It's estimated that the chance of two iris being identical is about 1 in $10^{78}$. The patterns are fixed from about one year in age and remain constant, apart from the occasional appearance of freckles and barring trauma, surgical procedures, and some rare diseases. The objective of this paper is not to explore the pattern recognition techniques that are used for iris identification, but to introduce a robust image processing technique to segment high quality images of the iris. 
The iris region in an image can be defined by two boundary circles, an inner circle that corresponds to the boundary of the pupil and iris, and an outer circle on the boundary of the iris and the sclera, called limbus. Figure 3 shows our 2D model of the iris, roughly defining the surfaces that must be segmented. Observe that the position of the upper and lower eyelids are arbitrary in this model, they may or may not occlude the iris, and, although the inner and outer circles of the iris are not concentrical, the distance between their centers is not significant in this model.

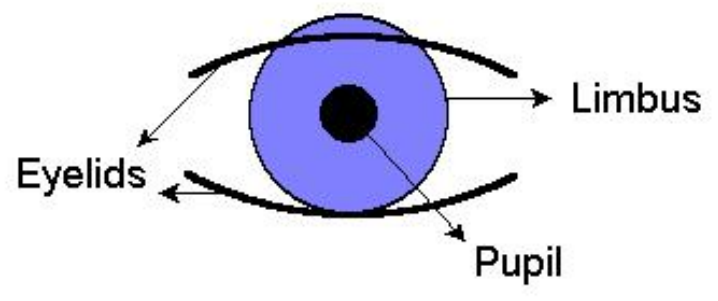

Figure 3. Iris region, defined by the inner (pupil) and outer (limbus) circles of the iris, and the upper and lower eyelids.

The segmentation of the boundaries of the iris must be reasonably accurate, so features can be reliably extracted and used for identification and authentication purposes. A variety of image processing techniques have been suggested for iris segmentation. The first step to guarantee a good image is to control the illumination conditions. The systems from Daugman [3] and Wildes et al. [9] use visible light and some apparatus to help the user finding the right position for image acquisition.

The use of near infrared (NIR) light between 700 and $900 \mathrm{~nm}$ has also been suggested, because of the advantage that NIR is practically invisible to the human eye. Figure 4 shows the geometry of the image acquisition using NIR light sources. The top image shows the general geometry of the camera (with center of projection $C$ ), eye (a sphere with center $E$ ), and one NIR point light source $L$. These 3 points in $3 \mathrm{D}$ space define a plane. Considering the spherical model of the eye, the corneal reflection $R$ is also contained in this plane. Now observe in the bottom image that, when a light source is placed near the optical axis of the camera, the points $C, L, R$ and $E$ are approximately collinear. One very important fact from this geometry is that, no matter where the eye is located in 3D, if we can detect the center of the pupil $P$, it is clear that when the eye is looking at the camera, then $P$ and $R$ are also collinear. This idea was explored in [1] to detect eye contact of the user with the pupil detector. When the eye is not looking at the camera, the corneal reflection $R$ may lie on the iris region. This situation must
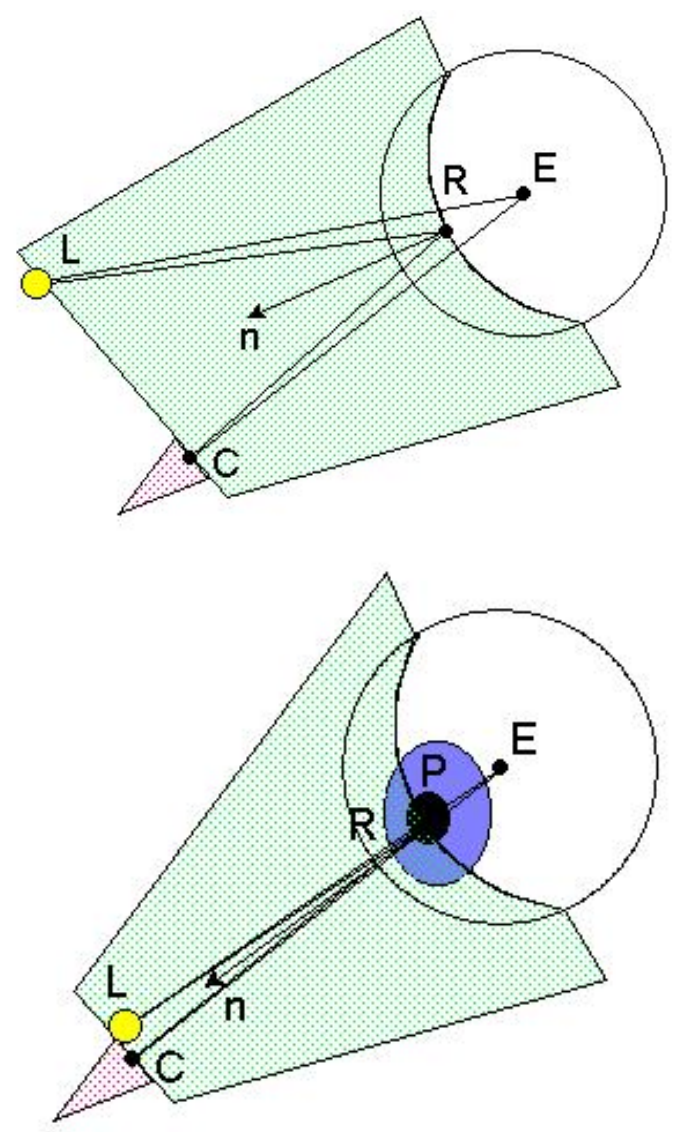

Figure 4. Geometry of the camera, eye and light sources. Observe, in the top image, the plane defined by the camera $C$, the light $L$ and the eye $E$. The cornea reflection $R$ lies on this plane. When $L$ is positioned close to the optical axis of the camera (bottom image), the points $C, L, R$ and $E$ are collinear.

be avoided by the image acquisition system to obtain clear images of the iris.

We propose the use of two NIR light sources to facilitate pupil detection and also reliably select the images that do not contain reflections on the iris region. Once the position and size of the pupil is computed, the iris and eyelids are segmented using a coarse-to-fine multiresolution strategy. Figure 5 shows the block diagram of our iris segmentation technique.

The dual NIR structural lighting scheme is similar to the eye tracking system described by Morimoto et al. [7]. Multiple light sources for pupil detection have also been suggested in $[8,4]$ to be used in eye gaze tracking applications. Figure 6 shows a picture of the setup of our camera and light sources. Observe the $L 1$ set of NIR LEDs are placed 


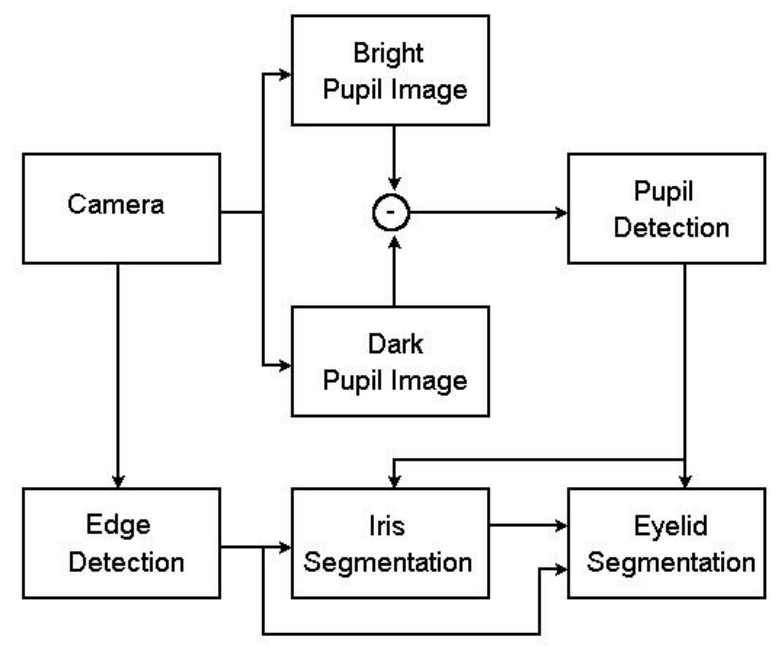

Figure 5. Block diagram of the iris segmentation system.

around the camera lens, as near as the optical axis as possible (on-axis light). The $L 2$ set of NIR LEDs (the second light source), is placed symmetrically, a few centimeters away from the optical axis of the camera (off-axis light).

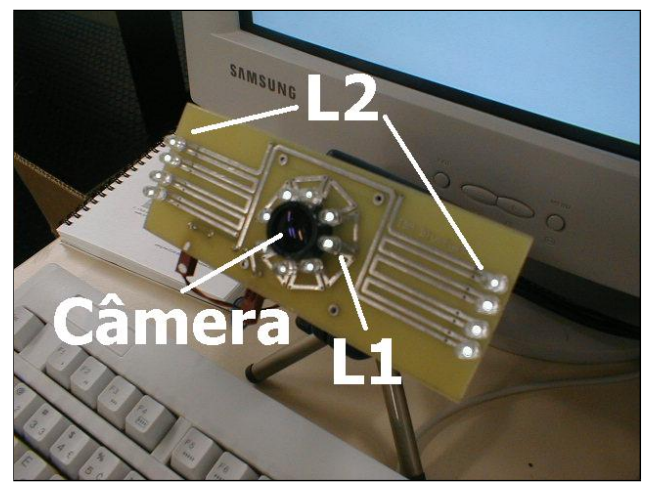

Figure 6. Camera with NIR light sources. L1 is placed near the optical axis of the camera to generate a bright pupil image, and L2 is placed away from the axis to generate a dark pupil image.

Each light is turned on and off alternately, synchronized with the video signal. When the on-axis light is on, the camera receives the light from the back of the eye (similar to the red eye effect commonly seen in flash photography), and the pupil appears bright. The off-axis light generates a dark pupil image, but the rest of the image remains at about the same brightness level. The high contrast region that corre- sponds to the pupil can be segmented from simple subtraction of the dark from the bright pupil image, as shown in Figure 7. The synchronization hardware is quite simple and can be easily constructed using off-the-shelf components.
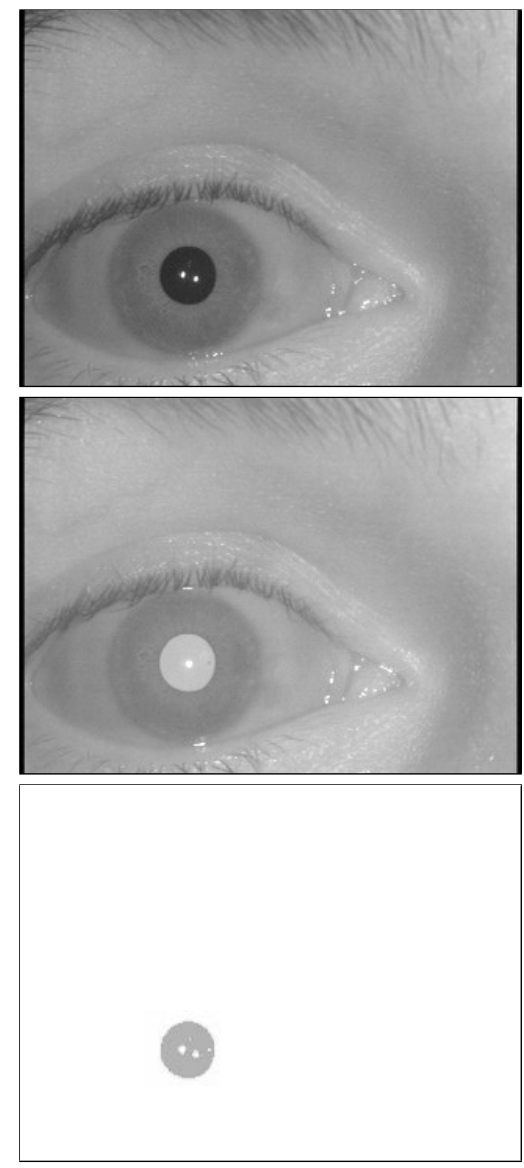

Figure 7. Dark, bright and difference pupil images.

The corneal reflection corresponds to a bright spot, that can be seen at the center of the pupils in Figure 7. Bright spots may appear on the corners of the eye, particularly when the eye is well lubricated with tears. Because we use the corneal reflections just to select good quality images, the algorithm simply searches for bright pixels near the center of the pupil region. When bright pixels are detected near the pupil center, the images are further processed to segment the limbus and eyelids, as described next.

\subsection{Segmentation of the limbus and eyelids}

Once the size and position of the pupil is computed from two consecutive frames of the video signal, the limbus and eyelids are segmented using a coarse-to-fine multiresolution strategy. Since the sclera is predominantly white, 
the gradient around the iris-sclera boundary points towards the sclera [5]. We use this fact to filter edge points that are used to fit the external circle around the iris.

Consider the horizontal and vertical Sobel masks shown in Figure 8. Since the iris can be occluded by the eyelids, the best edge points to fit the limbus circle are the vertical edges. Therefore, only the strong responses of the horizontal mask shown in Figure 8a are used to fit the limbus. We also know that the filter returns strong negative values on the left boundary region of the limbus, and strong positive values on the right boundary.

\begin{tabular}{|l|l|l|}
\hline-1 & 0 & +1 \\
\hline-2 & 0 & +2 \\
\hline-1 & 0 & +1 \\
\hline
\end{tabular}

(a)

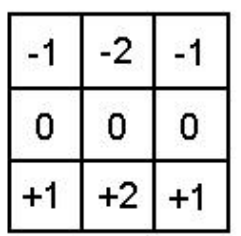

(b)

\section{Figure 8. Sobel convolution kernels used for gradient computation: a) horizontal and b) vertical kernels.}

We assume a similar hypothesis to fit ellipses for the eyelids, i.e., the best edge points for the eyelids are mostly horizontal, and therefore, only the strong responses of the vertical mask shown in Figure $8 \mathrm{~b}$ are used to fit the eyelids. We also know that the filter returns strong positive values for the upper eyelid, and strong negative values for the lower eyelid.

The iris segmentation starts by building a Gaussian pyramid of the input image. Edge detection using Canny's algorithm, that returns thin edges, is applied to all pyramid levels to create an edge pyramid. Examples of an image pyramid and an edge pyramid are shown in Figure 9. Observe that at the coarsest levels of the pyramid, the high frequency components that correspond to small details of the image such as noise and eyelashes are filtered out.

The search for the outer circle starts at the coarsest level of the edge pyramid, around a search box centered at the pupil. Edge points to the left with strong negative responses of the horizontal Sobel mask, and edge points to the right of the search box with strong positive responses are used to fit a circle. Other methods such as deformable templates [11] and Hough transform could also be implemented using these constraints, but this simple approach revealed quite robust and efficient during our experiments. Once the circle is estimated for the lowest pyramid resolution, it is refined using the next finer resolution pyramid level.

The eyelids are segmented in a similar coarse-to-fine strategy, after the segmentation of the iris. The position and
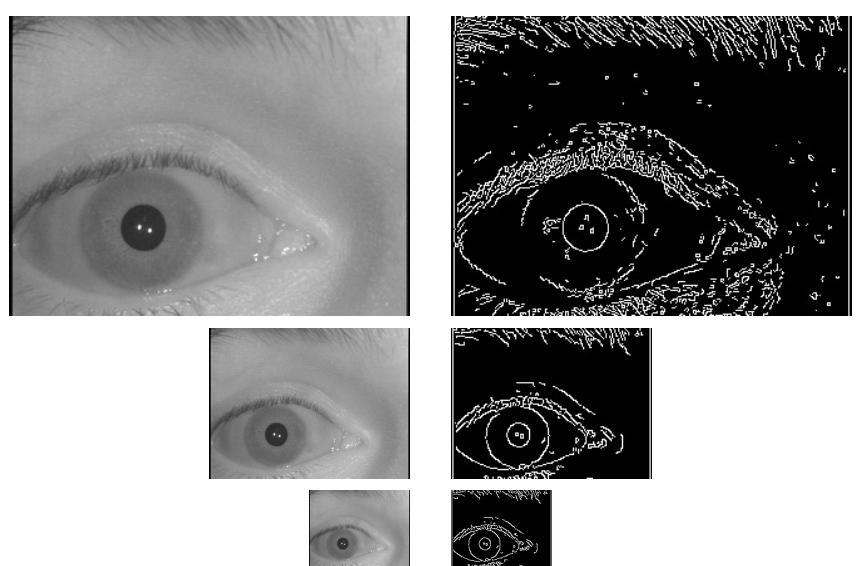

Figure 9. Image and edge pyramids.

size of the iris defines a search box for the upper and lower eyelids, at the lowest resolution of the edge pyramid. All pixels inside the iris are automatically discarded, and only the edge points with strong negative or positive responses from the vertical Sobel mask are used to fit the ellipses for the eyelids.

\section{Implementation issues and Experimental Results}

The following image qualities are desirable for iris recognition: frontal (instead of side views) high resolution images of the iris to avoid distortions, good focus and contrast levels to help feature extraction, and no extraneous (or as few as possible) bodies within the iris regions, such as eyelashes and specular reflections. Using the dual NIR active lighting technique for pupil detection, it is possible to automatically acquire images that satisfy most of these conditions.

Figure 10 shows some examples of pupils detected using the camera with active illuminators that are not appropriate for iris recogtion. Observe that the pupils are detected from quite arbitrary distances and orientations, and the system offers a very simple way of selecting appropriate images.

Good image contrast is achieved because of the NIR active illuminators. The illuminators are not powerful enough to cause harm, and are invisible to the human eye, so that the acquisition of the iris image does not cause any sort of discomfort to the user. The detection of eye contact (the corneal reflection is detected near the pupil center) guarantees that the corneal reflection is inside the pupil, therefore not corrupting the iris, and makes the acquisition process automatic. Another advantage of the method, due to the long focal length of the cameras being used, i.e., we can assume parallel projection, is that we have a nice frontal image of the iris. In case of perspective projection, the cen- 

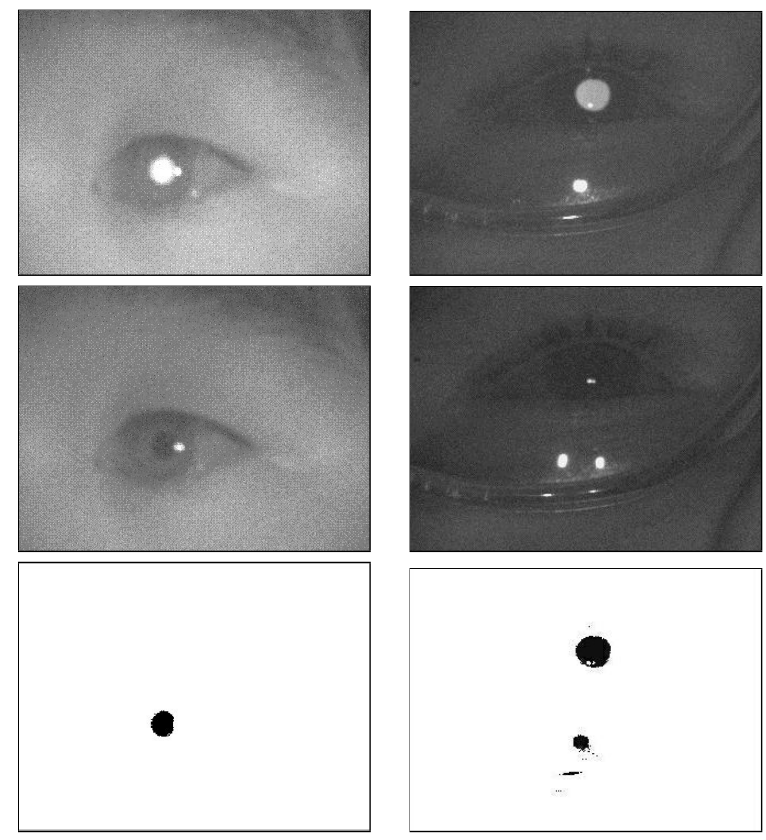

Figure 10. Images of eyes not looking at the camera.

ter of the pupil must also be near the center of the image (near the principal point of the camera), to avoid perspective distortions. Therefore the corneal reflection will always lie in the center of the pupil, and the iris pattern will not be corrupted by optical or perspective distortions. The system also works well with glasses, but eyelashes may constitute a problem.

The detection of the corneal reflection is simple because they are very bright pixels compared to the dark pixels of the pupil. The size of these reflections also gives an idea of the quality of the focus because, when the camera is in focus, the area of the reflections will be very small. To guarantee that the iris is completely within the image, another restriction can be defined for image acquisition, that the pupil center must be detected within a rectangle around the center of the image. The following constraints may be used to select appropriate images for iris recognition from the video stream:

- Presence of a pupil: hard to fool the system due to intrinsic properties of the eye;

- Presence of a corneal reflection near the center of the pupil: guarantees eye-contact with the camera, i.e., good image quality;

- Center of the pupil near the image center: reduces camera and perspective distortions;
- Corneal reflection with small area: guarantees that the image is in focus.

We have implemented a real-time prototype( 15 frames a second) of the pupil and corneal reflection detection and tracking system to automatically select good iris images on a Pentium III 1.5GHz platform running Linux. The user first presses a button and then positions her/his eye in front of the camera. The system automatically detects a good image, and alerts the user when the acquisition process has ended. Because we are using a fixed focus camera with a relatively small depth of field, the size of the iris is not constrained. Figure 11 shows a few results of the iris and eyelid segmentation algorithms. The contrast of the regions around the iris were enhanced using histogram equalization, so that the iris pattern can be easily seen. More results are available at http://latin.ime.usp.br/tts/ireyes.
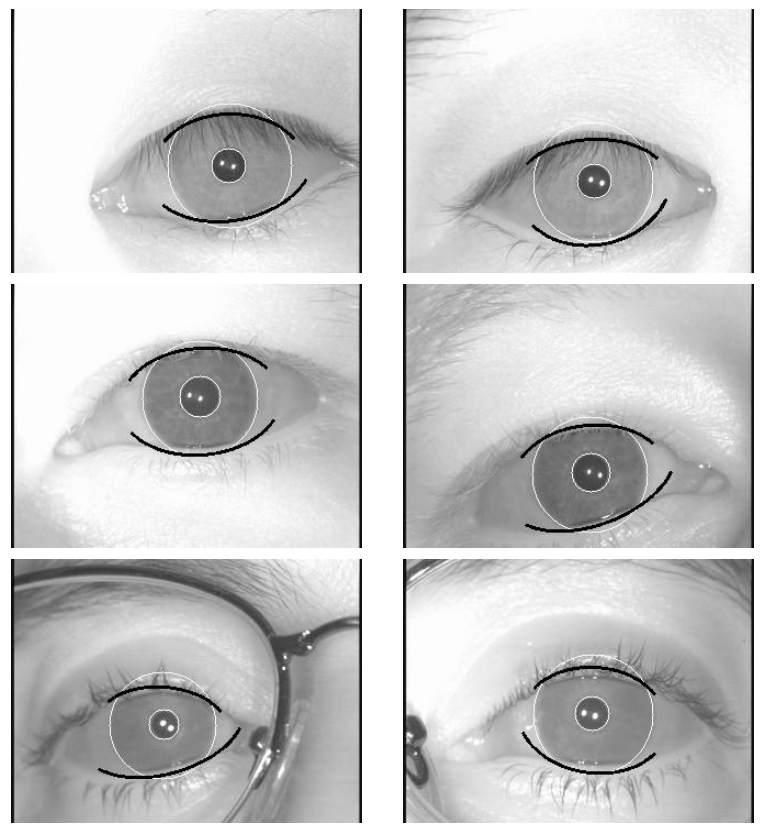

\section{Figure 11. Results from the pupil, iris, and eyelid segmentation.}

Other benefits of our system to biometric authentication is the additional security intrinsic to the dual light pupil detector. Because it relies on geometric properties and the red-eye effect, it is very hard to "fool" the system with a fake eye. Motion blur can also be avoided by computing the displacement of the pupil between frames.

\section{Conclusion}

We have presented a novel automatic iris segmentation technique based on a dual near infrared (NIR) illumination 
real-time pupil detector developed for eye gaze tracking. The pupil detector is very efficient and robust to changes in illumination conditions. Once the pupil position is computed, the iris and eyelids are segmented using a coarse-tofine strategy. The use of the dual NIR light sources presents several advantages, such as accuracy, robustness to illumination conditions, and easy identification of appropriate iris images. Good iris images for recognition are frontal to the camera, in focus, and without specularities over the iris region. Future work includes a interactive interface that will instruct the user how to find the correct position of his/her face in $3 \mathrm{D}$.

\section{References}

[1] A. Amir, D. Koons, M. Flickner, and C. Morimoto. Method and apparatus for determining eye contact. IBM Docket no. AM9-98-137 (patente requerida), December 1998.

[2] W. W. Boles and B. Boashash. A human identification technique using images of the iris and wavelet transform. IEEE Transactions on Signal Processing, 46(4):1185-1198, April 1998.

[3] J. Daugman. High confidence visual recognition of persons by a test of statistical independence. IEEE Transactions on Pattern Analysis and Machine Intelligence, 15(11):11481161, November 1993.

[4] Y. Ebisawa and S. Satoh. Effectiveness of pupil area detection technique using two light sources and image difference method. In A. Szeto and R. Rangayan, editors, Proceedings of the 15th Annual Int. Conf. of the IEEE Eng. in Medicine and Biology Society, pages 1268-1269, San Diego, CA, 1993.

[5] R. Kothari and J. Mitchell. Detection of eye locations in unconstrained visual images. In International Conference on Image Processing, volume I, pages 519-522, Lausanne, Switzerland, September 1996.

[6] L.Flom and A. Safir. Iris recognition system. U.S. patent 4641394, 1987.

[7] C. Morimoto, D. Koons, A. Amir, and M. Flickner. Pupil detection and tracking using multiple light sources. Image and Vision Computing, 18(4):331-336, March 2000.

[8] A. Tomono, M. Iida, and Y. Kobayashi. A tv camera system which extracts feature points for non-contact eye movement detection. In Proceedings of the SPIE Optics, Illumination, and Image Sensing for Machine Vision IV, volume 1194, pages 2-12, 1989.

[9] R. Wildes, J. C. Asmuth, G. L. Green, S. C. Hsu, R. J. Kolczynski, J. R. Matey, and S. E. McBride. A system for automated iris recognition. In IEEE Workshop on Applications of Computer Vision, pages 121-128, 1994.

[10] G. Wyszecki and W. Stiles. Color Science: Concepts and Methods, Quantitative Data and Formulae. John Wiley \& Sons, New York, 1982.

[11] A. Yiulle, P. Hallinan, and D. Cohen. Feature extraction from faces using deformable templates. International Journal of Computer Vision, 8(2):99-111, April 1992.
[12] Y. Zhu, T. Tan, and Y. Wang. Biometric personal identification based on iris patterns. In ICPROO, volume II, pages 805-808, 2000. 